\title{
INCONTINÊNCIA URINÁRIA EM IDOSOS: PROPOSTA PARA A CONDUTA DA ENFERMEIRA
}

Rosalina Aparecida Partezani Rodrigues* Maria Manuela Rino Mendes**

As auroras enfocam a Incontinência Urinária em idosos destacando a prevalência, suas causas e tipos. Subsidiam a avaliação do problema e a conduta, detalhando aspectos do histórico, exame físico, registro da incontinência bem como os exercícios para treinamento da bexiga, da musculatura, hábitos higiênicos bem como a manipulação de cateteres e aspectos do ambiente visando restaurar a continência e/ou tratar e conviver com a incontinência urinária. Destacam que a conduta da enfermagem se faz inter-relacionada com a equipe médica, com cuidadores, familiares e idosos, investindo no autocuidado para o convívio com a incontinência urinária e favorecendo a continência.

UNITERMOS: cuidado de enfermagem, incontinência urinária, idosos

\section{INTRODUÇÃO}

A incontinência é um problema de saúde significante para os idosos, não sendo considerada uma doença, mas uma condição que afeta essa parcela populacional em seus aspectos físicos e psicológicos, restringindo-Ihes a independência e a dignidade.

A Sociedade Internacional de Continência Urinária define incontinência urinária como "a condição onde a perda involuntária de urina é um problema social ou higiênico e objetivamente demonstrável", $\left(\mathrm{WYMAN}^{10}\right)$. Pesquisas recentes têm demonstrado que a incontinência não é um resultado do processo de envelhecimento no homem (CHENITZ et $\mathrm{al}^{1}$ ), mas cabe mencionar que existem mudanças relacionadas com o envelhecimento que

\footnotetext{
* Professor Doutor do Departamento de Enfermagem Geral e Especializada da Escola de Enfermagem de Ribeirão Preto - USP

** Professor Assistente do Departamento de Enfermagem Geral e Especializada a Escola de Enfermagem de Ribeirão Preto - USP
} 
contribuem para que este fato ocorra. Esta condição é causada por modificações específicas nas estruturas funcionais do corpo que freqüentemente resultam de doenças ou de uso de medicamentos. A incontinência é algumas vezes o primeiro e somente o único sintoma de infecção do trato urinário. Outras causas como a instabilidade do detrusor, a uretrite, a diabetes, as doenças do sistema nervoso central, a perda da cognição, entre outras, podem levar a incontinência urinária.

Essa alteração pode levar o idoso a experienciar problemas de ordem física, tais como: irritações da pele e infecções; psicossociais como a depressão, o isolamento social, a rejeição da família e a perda da confiança em si e econômicos, pois vai exigir cuidados que implicarão gastos com a hospitalização (RODRIGUES; ZAGO).

Esta temática tem recebido pouca atenção na literatura médica e de enfermagem. $A$ avaliação clínica e urologia do médico e da enfermeira são requisitos essenciais para se identificar as causas de tal problema, bem como o diagnóstico das condições neurológicas, urologias e psíquicas do idoso. Para o trabalho da enfermeira gerontológica e geriátrica é fundamental que a mesma conheça a prevalência, causas e tipos da incontinência urinária para planejar a conduta para a intervenção de enfermagem, objetivando promover a continência e facilitar o convívio do idoso com essa alteração.

\section{PREVALÊNCIA DA INCONTINÊNCIA URINÁRIA}

No que se refere a prevalência da incontinência no idoso, verifica-se através da revisão da literatura que não há um registro nos dados, nos estudos populacionais, e definições de incontinência (CHENITZ et al. ${ }^{1}$ ). Em geral as pesquisas demonstram que as mulheres são afetadas duas vezes mais do que os homens, nessa condição.

A prevalência estimada é de $5 \%$ a $37 \%$ para idosos que vivem na comunidade, $38 \%$ a 55\% para os que estão institucionalizados por tempo prolongado e $19 \%$ para idosos em hospitais de cuidado a episódios agudos (MOHIDE ${ }^{5}$; DIOKNO, BROCK, BROWN et al. ${ }^{2}$ ).

O estudo de SOLOMON ${ }^{9}$ sobre incontinência urinária em idosos, apresenta prevalência de $10 \%$ a $20 \%$ naqueles que vivem na comunidade, $19 \%$ a $35 \%$ em hospitalizados é em torno de 50\% nos lares, residências de enfermagem, tipo asilo.

Apesar das diferenças apresentadas na prevalência de diversos estudos na literatura, observa-se que a condição da incontinência urinária é relativamente freqüente na população que envelhece. 


\section{CAUSAS E TIPOS DE INCONTINÊNCIA URINÁRIA}

Para se avaliar a incontinência urinária é necessário distinguir os seus dois tipos: a aguda ou transitória e a persistente ou estabelecida.

A incontinência aguda ou transitória está basicamente associada a uma condição clínica ou cirúrgica aguda e freqüentemente é resolvida quando as causas que a predispõem são eliminadas $\left(\mathrm{WYMAN}^{10}\right.$ \}. De acordo com KANE, OUSLANDER e ABRASS ${ }^{3}$ as causas da incontinência aguda ou transitória podem ser:

01. delirium - a existência do estado confusional do idoso chega às vezes a interferir na capacidade de se dirigir ao sanitário em tempo para o esvaziamento vesical;

02. restrição da modalidade - onde a freqüência e urgência miccional estão presentes;

03. infecção, inflamação - do trato urinário ou sistêmica, que favorecem o aparecimento da incontinência;

04. medicamentos, psicológica - onde os sedativos, diuréticos, relaxantes musculares, narcóticos e anticolinérgicos, podem contribuir para o desenvolvimento da incontinência e fatores psicológicos como a hostilidade, raiva, depressão também podem ocasionalmente estar associados a ela.

A incontinência persistente ou estabelecida pode ter aparecimento súbito apresentando quadro agudo, ou desenvolver-se gradualmente, sem se conhecer a causa que a precipita, freqüentemente evoluindo com severidade $\left(\mathrm{WYMAN}^{10}\right)$.

Este tipo de incontinência pode ser classificado em quatro categorias discriminadas por WYMAN ${ }^{10}$, como se segue:

01. incontinência de stress - é a perda involuntária da urina quando a pressão intravesical excede a pressão uretral máxima, devido a elevação da pressão intrabdominal e ausência da contração do detrusor. Este tipo descreve a perda da urina durante exercícios, ou em situações de espirro ou riso. É mais freqüente em mulheres, mas pode ocorrer em homens após cirurgia de próstata;

02. "urge" incontinência - é a perda involuntária da urina associada ao forte desejo de urinar, freqüentemente associada com a condição da pessoa, como no caso de Acidente Vascular Cerebral, Demência Senil, Doença de Parkinson, Escleroses Cerebrais Múltiplas, podendo ocorrer também em pessoas idosas saudáveis. Está associada com a instabilidade do músculo detrusor, inibição e hiperreflexia da bexiga;

03. incontinência de superfluxo - é a perda involuntária da urina quando a pressão intravesical excede a pressão máxima da uretra devido a elevação da primeira associada 
a distensão da bexiga na ausência de atividade de detrusor. As possíveis causas incluem: obstrução anatômica devido ao aumento da próstata; bexiga neurogênica não inibida, resultante da perda da função iniciadora sobre a micção, quando o idoso percebe que a bexiga está distendida, mas não consegue inibir o reflexo miccional, apresentando perdas freqüentes de pequeno volume de urina; bexiga neurogênica atônica em que o indivíduo não percebe a distensão vesical com o acúmulo de urina e, portanto, o reflexo miccional não chega a ser desencadeado, como ocorre nos pacientes diabéticos;

04. incontinência funcional - é diagnosticada quando há perda involuntária da urina associada a incapacidade ou falta de vontade de usar o toalete apropriadamente. Está associada às perdas cognitivas e físicas dos idosos e a fatores psicológicos e ambientais que influenciam o uso da toalete.

\section{CONDUTA DA ENFERMEIRA EM IDOSOS COM INCONTINÊNCIA URINÁRIA}

Baseado em nossas experiências enquanto enfermeiras assistenciais e docentes da área de Enfermagem Gerontológica e Geriátrica podemos afirmar que a enfermeira assiste aos idosos tanto em hospitais, em diversas instituições prestadoras de cuidado a saúde, bem como no domicílio; portanto o conhecimento sobre o impacto da incontinência urinária na população que envelhece, seus tipos e causas são aspectos fundamentais a considerar na avaliação do problema e subseqüente conduta.

A avaliação clínica da incontinência destaca as disfunções do sistema urinário e seus efeitos nos demais sistemas corporais; e um enfoque específico do médico para determinar o diagnóstico. A avaliação da enfermeira parte dos aspectos fisiológicos sem, entretanto, deixar de enfocar os aspectos psicológicos, sociais e ambientais que influenciam o quadro do problema, portanto introduz variantes ao modelo clínico, com destaque psicossocial.

A avaliação realizada pela enfermeira está voltada para orientar a conduta de reestruturação da continência sempre que possível, ou facilitar o convívio com a incontinência, quer no contexto institucional ou na comunidade e enfoca o idoso em seu contexto sócio familiar.

CHENITZ et al. ${ }^{1}$ e NELSON ${ }^{6}$, sugerem uma avaliação diagnóstica do médico e da enfermeira, para detectar incontinência urinária a partir da história, exame físico do paciente e guias de manuseio para o treinamento da bexiga e do hábito de urinar (anexo 1, 3, 4, 5).

A história do problema deve procurar a investigação de informações 
para descrever o quadro, detalhando o seu início, duração, freqüência, período, quantidade e características da urina, sintomas, hábitos higiênicos, alimentares e de hidratação. A caracterização das habilidades para o autocuidado engloba a história geniturinária, a neurológica, e clínica em geral, além da psicológica, o ambiente, as medicações, os cuidados básicos e a autopercepção sobre a incontinência urinária.

O exame físico envolve a descrição da função neurológica, o exame abdominal, genital e retal.

A avaliação funcional da incontinência urinária pode ser facilitada pelo registro regular das micções sugerido por OUSLANDER et al. ${ }^{7}$ (anexo 2), quando o idoso se encontra institucionalizado e pode ser adaptado ao domicílio desde que se delegue a alguém a execução da monitorização do controle miccional. Os registros têm o propósito de documentar o estado da continência, os fatos associados com os episódios da incontinência, o planejamento e a monitorização do treinamento da bexiga, o treinamento de hábitos, o programa de horário para usar o toalete, o seguimento objetivo dos resultados da conduta. A avaliação inclui também os dados laboratoriais e de avaliação urodinâmica (que detecta a alteração da bexiga e uretra), para identificar o estado de incontinência (RODRIGUES; ZAGO ${ }^{8}$ ).

O tratamento do idoso incontinente deve ser realizado pela equipe de saúde e com a participação do paciente e das pessoas que Ihes prestam cuidados. O trabalho da enfermeira é identificar a incontinência urinária em idosos, planejar as estratégias de intervenção aplicá-las e avaliar os resultados. Sua meta é prevenir novos casos de incontinência, melhorar a condição dos idosos incontinentes através de cuidados básicos de higiene, da educação para o controle miccional e da prevenção de lesões no períneo e favorecer um ambiente adequado, para manter a continência, conservando o idoso seco evitando dessa forma outras complicações.

Tomando por base os dados da história sócio-familiar do idoso, o exame físico e a consulta a registros no prontuário sugerido por $\mathrm{CHENITZ}^{1}$ e $\mathrm{NELSON}^{6}$, a enfermeira fará uma análise cuidadosa objetivando:

\section{1. caracterizar o problema;}

02. traçar a conduta para idosos incontinentes, que podem desenvolver o controle urinário e idosos incontinentes, que não podem ter esse controle e vão exigir suporte familiar e da enfermagem;

03. definir os resultados esperados para as estratégias de ação;

04. determinar as estratégias de enfermagem que podem ser orientadas para:

04. a) a terapêutica medicamentosa - freqüentemente usada em combinação com outras terapias, onde a enfermeira tem o papel principal de implementá-las:

- instruir o paciente quanto ao uso do medicamento, horários, quantidade e via de administração; 
- orientar os idosos e familiares quanto aos resultados da medicação;

- observar os resultados (tanto os efeitos esperados quanto os indesejáveis);

- manter o registro da freqüência da incontinência urinária;

- registrar mudanças da pressão arterial, do estado mental e das habilidades funcionais;

- alertar o médico sobre os resultados;

04. b) a terapêutica cirúrgica - indicada em alguns pacientes idosos incontinentes, de acordo com o diagnóstico médico. O trabalho da enfermeira nesta estratégia consiste em promover o cuidado e educação do paciente e família no período pré e pós-operatório quanto aos: resultados esperados; anestesia e ao procedimento cirúrgico; dor e outros desconfortos e sintomas; período perioperatório, incluindo cateterização; alta hospitalar; limitações da atividade sexual; complicações pós-operatórias;

04. c) os procedimentos de treinamento para o controle urinário - o idoso incontinente vai ser manuseado, tanto pela família, quanto pelos que Ihe prestam cuidados. As medidas terapêuticas podem ser utilizadas nas estratégias da enfermeira, isoladamente e/ou em combinação com medicamentos e cirurgias. Entre eles destacamos os exercícios de Kegel, treinamento da bexiga e do hábito miccional e o biofeedback (CHENITZ et al. ${ }^{1}$ ), que podem ser utilizadas como opção na conduta de incontinência urinária:

04. c.1 - exercícios de "Kegel" - o objetivo dos mesmos é fortalecer os núcleos pélvico, vaginal e ileococcígeo. Podem ser realizados em idosas que têm função cognitiva presente, musculatura da parede pélvica adequada e motivação para seguir o procedimento (anexo 3);

04. c.2 - treinamento da bexiga e do hábito - o objetivo é restaurar a função normal da bexiga pela resistência a sensação de urgência. É um método pelo qual o indivíduo incontinente gradualmente estende seu intervalo de micção para o padrão normal. 0 treinamento do hábito, visa a modificar a função da bexiga é manter o paciente seco. Deve ser seguido um esquema rígido de intervalos curtos de tempo e útil na incontinência tipo "urge" e funcional (anexo 4).

LONG $^{4}$ sugere quatro intervenções da enfermeira nesse procedimento: manter a entrada de fluído adequada; identificar o método mais apropriado de usar a toalete ou fazer higiene; oferecer um horário para o esvaziamento vesical e higiene apropriada e manter o registro da incontinência;

04. c.3 - "biofeedback" - é uma terapia comportamental cujo objetivo visa alterar a resposta fisiológica da bexiga e dos músculos do assoalho pélvico de forma a manter a continência. Deve-se ensinar o paciente a inibir voluntariamente as contrações da bexiga ou contrair os músculos esfincterianos para prevenir o esvaziamento da mesma. É um procedimento adequado para incontinência de stress ou "urge". 
As respostas esperadas as condutas consistem no decréscimo de episódios de incontinência urinária e de complicações desta, bem como melhora no seu manejo, aspectos estes que se devem considerar na avaliação do uso do método;

\section{4. c.4 - utilização de cateteres internos e externos para manejar a incontinência} urinária. A cateterização é um dos tratamentos mais controvertidos na incontinência urinária. Os três tipos básicos de cateteres são: sonda vesical de demora, cateter intermitente externo.

Para se utilizar a cateterização interna é fundamental a avaliação médica e de enfermagem para se determinar o tipo de incontinência e o local em que o idoso vive. Devese avaliar a escolha da cateterização, considerando-se também as complicações que poderão advir, como infecções do trato urinário.

A cateterização intermitente deve ser avaliada quanto aos seus benefícios; é uma alternativa que deve ser utilizada em pacientes idosos que têm boa visão, habilidade manual, capacidade cognitiva íntegra ou ainda se a família e outros cuidados puderem aprender e se estiverem motivados a procedimentos rigorosos de assepsia para prevenir infecções vesicais.

Outro tipo de opção é o sistema de coletor externo, porém só oferece vantagens para o homem devido a anatomia do órgão genital, não se aplicando às mulheres (anexo 5);

\section{4. c.5 - planejamento da organização ambiental.}

A organização ambiental em que o idoso incontinente vive contribui para o seu tratamento. Atenção deve ser prestada às barreiras ambientais mais próximas, à luz suficiente e a não colocação de materiais em excesso no toalete. Outros aspectos a serem considerados é o uso de recipientes coletores de urina e a acomodação em cadeiras.

\section{CONSIDERAÇÕES FINAIS}

A incontinência urinária é um dos problemas silenciosos na geriatria, associado ao declínio filosófico e funcional da pessoa idosa, e muitas vezes negligenciado pelos profissionais da saúde. Assim, este trabalho apresenta uma proposta para a conduta da enfermeira frente à incontinência urinária. $\mathrm{O}$ médico é responsável em diagnosticar o tipo de incontinência urinária, condições fisiopatológicas e a prescrição medicamentosa para tratá-la ou controlá-la, e a enfermeira por avaliar juntamente com a equipe de saúde, familiares e o próprio idoso o quadro da incontinência. Presta cuidados no controle e no apoio da higiene e na prevenção de complicações, bem como 
na implementação das diversas estratégias para restaurar a continência urinaria. A educação continuada da enfermeira deve-se estender para o conhecimento e as habilidades da avaliação, e estratégias do idoso incontinente.

\section{URINARY INCONTINENCE IN ELDERLY PEOPLE: A PROPOSAL FOR NURSE'S CONDUCT}

The authors make it evident the Urinary Incontinence in elderly people showing up the prevalence, their motives and types. It is subsidize the evaluation of the historical. the physical exam, registry of the incontinence and also the exercises for the training of the bladder, the musculature, hygiene habits and also the manipulation of the catheters and the aspects of the environment looking for the restoration of the continence and/or to treat and to live with in the urinary incontinence. They distinguish that the nursing conduct is done interrelated with the medical team with homely cares and elderly investing in the self care for to improve the urinary incontinence and favoring the continence.

UNITERMS: nursing care, urinary incontinence, elderly

\section{INCONTINENCIA URINARIA EN ANCIANOS: PROPUESTA PARA CONDUCTA DE LA ENFERMERA}

Las autoras enfocan la incontinencia urinaria en ancianos, destacando la prevalecía, las causas y tipos. Proporcionan elementos para evaluar el problema y la acción profesional, detallando aspectos de la historia del paciente. Se centran sobre el examen físico y la incontinencia urinaria, as (como en los ejercicios para el entrenamiento de la bejiga y refuerzo de la musculatura interveniente. También, informan sobre hábitos higiénicos y manipulación de catéteres, incluyendo los aspectos ambientales, buscando restaurar la continencia, tratarla y si es el caso, convivir con la incontinencia urinaria. Destacan aún, que la acción de enfermería se hace en interacción con el equipo médico, con cuidadores, familiares y ancianos, teniendo en mente la inversión en el auto cuidado.

UNITERMOS: cuidado de enfermería, incontinencia urinaria, ancianos 


\section{REFERÊNCIAS BIBLIOGRÁFICAS}

01. CHENITZ, W. C., STONE, V. T., SALISBURY, S.A. Clinical gerontological nursing: a guide to advanced practice. Philadelphia: W. B. Saunders, 1991.

02. DIOKNO, A. C., BROCK, B. M., BROWN, M. B. et al. Prevalence of urinary incontinence and other urological symptoms in the noninstitutionalized elderly. J. Urol., v. 136, p. 1022-25, 1986.

03. KANE, R. L., OUSLANDER, J. G., ABRASS, I. B. Essentials of clinical geriatrics. New York: Mc Graw Hill Book, 1984.

04. LONG, M. L. Incontinence: defining the nursing role. J. Gerontol. Nurs., v. 11, n. 1, p. 30-41, 1985.

05. MOHIDE, E. A. The prevalence and scope of urinary incontinence. Clin. Geriatr. Med., v. 2, p. 639-56, 1986.

06. NELSON, J. D. Urinary incontinence. In: ROGERS-SEIDL, F. F. Geriatric nursing care plans. St. Louis: Mosby Year Book, 1991. p. 274-80.

07. OUSLANDER, U. G., URMAN, H. N., UMAN, G. Development and testing of and incontinence monitoring record. JAGS, v. 34, n. 2, p. 83-90, Feb, 1986.

08. RODRIGUES, R.A.P., ZAGO, M.M.F. Incontinência urinária no idoso: assistência de enfermagem. Rev.Esc.Enf.USP, v. 25, n. 1, p. 21-8, Abri11991.

09. SOLOMON, D.H. New issues in geriatric care. Ann. Intern. Med., v. 108, n. 5, p. 718-32, 1988.

10. WYMAN, J.F. Nursing assessment of the incontinent geriatric out patient population.

Nurs. Clin. North. Am., v. 23, n. 1, p. 169-87, 1988. 


\section{ANEXO 1}

\section{HISTÓRIA DO PACIENTE}

\section{CARACTERÍSTICAS DA INCONTINÊNCIA}

. início, duração e freqüência

. período (diurno, noturno ou ambos)

- quantidade e tipo de perda

. circunstâncias em que ocorre a incontinência

. dor

. sintomas associados a incontinência (urgência, gotejamento após ato de urinar, sensação de esvaziamento incompleto da bexiga)

. uso de tampões (número de tampões ou mudanças de roupas por dia)

. estratégias de condutas no passado e atualmente (treinamento da bexiga, exercícios de parede pélvica, terapia medicamentosa, cirurgia e outros)

. padrões de toalete normal

. diurno/noturno, freqüência, horário

\section{CARACTERÍSTICAS DA URINA}

. cor

. odor

. aparência

. $\mathrm{pH}$

- glucose

. proteínas

. acetonas

\section{ENTRADAS E SAÍDAS DE LÍQUIDOS}

\section{NUTRIÇÃO E HIDRATAÇÃO}

. peso ideal

. peso atual

. nutrientes ingeridos: adequacidade e padrões

. hidratação: quantidade, tipo e padrão

\section{HABILIDADES DE AUTO CUIDADO}

5.1. Cuidados básicos

. troca de roupas

. deambulação para toalete

. bengalas, andadores, barreiras, corrimões, distância para toalete 
. deficiências visuais ou auditivas

.tolerância a atividade

\subsection{História Geniturinária}

. enurese na infância

. mulheres - filhos (números, partos traumáticos)

. cirurgias anteriores (pélvica ou trato urinário baixo)

. infecções periódicas do trato urinário

. câncer da bexiga

. doença renal

5.3. História Neurológica

. acidentes cerebrovascular

. doença de Parkinson

. demência

- História Médica Geral

. doenças agudas

. diabetes mellitus

. doenças cardiovasculares (hipertensão, falência cardíaca congestiva)

. desordens intestinais (constipação, incontinência fecal)

\subsection{História Psicológica}

. estado emocional presente (nível de ansiedade, depressão, sentimentos

sobre a hospitalização)

. funções cognitivas (memória de longo e curto tempo), orientações

- julgamento

. auto descrição da história de vida

. percepção de saúde

. conhecimento do problema e tratamento

- sistema de suporte

. fatores de risco (fumo, bebida, auto-medicação)

\subsection{Medicação}

. incluindo medicação prescrita e não prescrita

5.6. Percepção dos cuidados e dos pacientes

- percepção da causa e severidade

.interferência das atividades da vida diária

. motivação para a continência

. expectativa de cura 
5.7. Características Ambientais

. acessibilidade a toalete

. distância da toalete

. necessidade e uso da toalete

\section{EXAME FÍSICO}

6.1. Exame Neurológico

. estado mental

. humor

. sinais de doença de Parkinson

. modo de andar: marcha e balanço do corpo

6.2. Exame Abdominal

. bexiga distendida

. desconforto suprapúbico

. massas

6.3. Exame Genital

.condições da pele

. sinais de infecção

. reflexo bulbocavernoso

. mulheres - vaginite atrófica, diminuição pélvica

6.4. Exame Retal

. tônus esfincteriano

. impactação fecal

. massas

. homens/dilatação prostática

6.5. Outros 


\section{ANEXO 2 \\ REGISTRO DA INCONTINÊNCIA URINÁRIA}

Instruções: cheque cada tempo do paciente. Marque no registro abaixo:

\begin{tabular}{ll} 
IPQ = incontinente, pequena quantidade & $\mathrm{s}=$ seco \\
$\mathrm{IGQ}=$ incontinente, grande quantidade & $\mathrm{UC}=$ urinou corretamente $(\mathrm{em} \mathrm{ml})$ \\
\hline NOME DO PACIENTE _ LEITO & BEXIGA
\end{tabular}

\begin{tabular}{|c|c|c|c|c|}
\hline Horário & $\begin{array}{l}\text { Incontinência } \\
\text { da Urina }\end{array}$ & SECO & $\begin{array}{l}\text { URINOU CORRE- } \\
\text { TAMENTE }\end{array}$ & COMENTÁRIOS \\
\hline
\end{tabular}

\begin{tabular}{|c|c|}
\hline 12 & $\mathrm{ml}$ \\
\hline 13 & $\mathrm{ml}$ \\
\hline 14 & $\mathrm{ml}$ \\
\hline 15 & $\mathrm{ml}$ \\
\hline 16 & $\mathrm{ml}$ \\
\hline 17 & $\mathrm{ml}$ \\
\hline 18 & $\mathrm{ml}$ \\
\hline 19 & $\mathrm{ml}$ \\
\hline 20 & $\mathrm{ml}$ \\
\hline 21 & $\mathrm{ml}$ \\
\hline 22 & $\mathrm{ml}$ \\
\hline 23 & $\mathrm{ml}$ \\
\hline 24 & $\mathrm{ml}$ \\
\hline 01 & $\mathrm{ml}$ \\
\hline 02 & $\mathrm{ml}$ \\
\hline 03 & $\mathrm{ml}$ \\
\hline 04 & $\mathrm{ml}$ \\
\hline 05 & $\mathrm{ml}$ \\
\hline 06 & $\mathrm{ml}$ \\
\hline 07 & $\mathrm{ml}$ \\
\hline 08 & $\mathrm{ml}$ \\
\hline 09 & $\mathrm{ml}$ \\
\hline 10 & $\mathrm{ml}$ \\
\hline 11 & $\mathrm{ml}$ \\
\hline
\end{tabular}




\section{ANEXO 3}

\section{EXERCÍCIO DE "KEGEL”}

Encontre o seu músculo Pubococcígeo (PC)

1. Pratique parando sua urina na metade do caminho

2. Para sentir o PC trabalhando insira o periômetro ou o dedo parcialmente na vagina enquanto é contraído

3. Se o seu abdômen e as nádegas movem, você está usando os músculos erradamente.

Desempenho do Exercício

1. Contraia seu músculo PC e segure-o por três segundos, então solte por mais três segundos. Repita este exercício mais de dez vezes (em 1 minuto)

2. Contraia seu músculo PC muito rapidamente, então solte. Tente fazê-lo o mais que puder durante 1 minuto.

Tempo do Exercício

1. Realize em 2 minutos a rotina 3 vezes/dia

2. Desde que ninguém esteja vendo, você tem a vantagem de fazê-lo em um tempo livre - durante o período de folga do trabalho, enquanto aguarda o ônibus, etc. 


\section{ANEXO 4 \\ GUIA PARA ENFERMEIRA MANUSEAR O TREINAMENTO \\ DA BEXIGA E DO HÁBITO}

\section{Intervenções}

1. Expor o plano ao paciente e família e aguardar o resultado. Conceder 6 semanas para a experimentação.

2. Incluir o plano de cuidado a entrada de fluídos $(2.000 / 3.000 \mathrm{ml} / 24$ horas), utilizar estratégias, para ir ao toalete e a manutenção do registro de incontinência.

3. Controlar a incidência da incontinência e modificar o plano da toalete cada 24 horas.

4. Prever um feedback positivo ao paciente, o mais apropriado.

5. Registrar os resultados para equipe de saúde e modificar o plano de cuidados de acordo com o uso de drogas, outros diagnósticos, planos de alta.

\section{Guia de Ensino ao Paciente}

1. Esperar o resultado do treinamento.

2. Plano de treinamento, método de toalete, entrada de líquido, horário de urinar.

3. Tipo, quantidade e tipo de entrada dos líquidos.

4. Explicação das drogas usadas em combinação com programa de treinamento.

5. Discussão a respeito das dores ou de novos problemas que podem aparecer durante o treinamento, distensão da bexiga, constipação, e quando deve ser registrado. 


\section{ANEXO 5 \\ CATÉTERES (EXTERNOS, INTERNOS) PARA MANEJAR A INCONTINÊNCIA URINÁRIA}

Intervenções

1. Esclarecer o propósito, resultados da cateterização com o paciente e família.

2. Registrar os sinais e sintomas de infecção ou obstrução do cateter.

3. Modificar o vestuário para acomodar os dispositivos dos cateteres e permitir mobilidade, como mais apropriado.

4. Avaliar o que esta sendo feito a respeito da cateterização como a opção mais segura e apropriada.

\section{Guia de Ensino ao Paciente e familiares}

1. Cuidados como o cateter, isto é, limpeza e manuseio.

2. Auto-aplicação.

3. Cuidado com o equipamento, isto é, limpeza do cateter e do coletor.

4. Prescrição de entrada de líquidos (2000/3000 ml/24 horas).

5. Sinais e Sintomas de complicações isto é, infecção, obstrução, dano no cateter.

\section{Critério do resultado para avaliação}

O paciente deve ter manejo da incontinência urinária sem complicação de infecção ou dano.

Agradecemos ao Dr. Jeová Nina Rocha, pelas sugestões feitas neste artigo. 